\title{
FLUTUAÇÕES TEMPORAIS NOS PADRÕES DE DISTRIBUIÇÃO DIAMÉTRICA DA COMUNIDADE ARBÓREO-ARBUSTIVO E DE 15 POPULAÇÕES EM UM FRAGMENTO FLORESTAL ${ }^{1}$
}

\author{
Evandro Luiz Mendonça Machado², Anne Priscila Dias Gonzaga², Warley Augusto Caldas Carvalho², \\ Josival Santos Souza ${ }^{4}$, Pedro Higuchi ${ }^{5}$, Rubens Manoel dos Santos ${ }^{3}$, Ana Carolina da Silva ${ }^{5}$ e Ary \\ Teixeira de Oliveira Filho ${ }^{6}$
}

\begin{abstract}
RESUMO - Objetivando caracterizar possíveis flutuações nos padrões de distribuição diamétrica foram analisadas, num intervalo de cinco anos (2000-2005), a comunidade e as populações das 15 espécies mais abundantes do compartimento arbóreo de um remanescente de Floresta Semidecídua. A distribuição diamétrica da comunidade apresentou alta concentração nas classes menores e diminuição acentuada no sentido das maiores (J-invertido), em ambos os inventários. As populações apresentaram padrões distintos, arbitrariamente distribuídos em dois grupos: Grupo 1, formado por espécies de menor porte e abundantes no sub-bosque, como Galipea jasminiflora, Allophylus edulis, Sebastiania commersoniana, Dendropanax cuneatus e Mollinedia widgrenii, e também espécies de árvores altas e pioneiras, como Acacia polyphylla e Piptadenia gonoacantha, cujas distribuições diamétricas seguiram o modelo J-invertido. Com exceção de Calycorectes acutatus, todas as demais espécies do Grupo 2 (Platycyamus regnellii, Cupania vernalis, Machaerium stipitatum, Machaerium villosum, Copaifera langsdorffii, Persea major e Cassia ferruginea) eram de maior porte, cujos adultos predominavam no dossel da floresta, elevando a área basal e apresentando densidade de indivíduos menor nas classes diamétricas inferiores. No intervalo considerado, apenas $D$. cuneatus alterou seu padrão de distribuição (Grupo 1-Grupo 2), enquanto as demais os acentuaram. No Grupo 1, a síndrome de dispersão preferencial era anemocórica ou autocórica, ao passo que no Grupo 2 predominava a zoocoria. Assim, o remanescente, por apresentar área pequena, forma alongada e localização semiurbana associada a um regime de intensas perturbações, pode ter ocasionado empobrecimento da fauna dispersora, prejudicando a taxa de recrutamento.
\end{abstract}

Palavras-chave: Floresta Estacional Semidecidual, Comunidade arbóreo-arbustiva, Dinâmica florestal e Estrutura diamétrica.

\section{TEMPORAL FLUCTUATIONS IN THE DIAMETRIC DISTRIBUTION PATTERNS OF THE TREES AND SHRUB COMMUNITY AND 15 POPULATIONS IN A FOREST FRAGMENT}

\begin{abstract}
Aiming to characterize possible fluctuations in the patterns of diametric distribution, the community and the populations of the 15 most abundant tree species in a fragment of semideciduous forest were analyzed in a five year interval (2000-2005). The diametric distribution of the community showed a high concentration in the smaller classes and accentuated reduction in the biggest classes (reverse-J-shape), for both surveys. The populations presented different patterns, randomly distributed in two groups. Group1, formed by species of smaller diameters and abundant in the sub-canopy, such as Galipea jasminiflora, Allophylus edulis, Sebastiania commersoniana, Dendropanax cuneatus and Mollinedia widgrenii, and also by pioneer species of high trees, Acacia polyphylla and Piptadenia gonoacantha followed the reverse-J-shape curve diametric distributions . All species of Group2 (Platycyamus regnellii, Cupania vernalis, Machaerium stipitatum, Machaerium villosum,
\end{abstract}

\footnotetext{
${ }^{1}$ Recebido em 28.09.2007 e aceito para publicação em 02.03.2010.

${ }^{2}$ Universidade Federal dos Vales do Jequitinhonha e Mucuri, UFVJM, Brasil. E-mail: <machadoelm@gmail.com>e<diaspri@gmail.com>.

${ }^{3}$ Universidade Federal de Lavras, UFLA, Brasil. E-mail: <wacaldas@yahoo.com.br> e <santosfloracaatinga@yahoo.com.br>.

${ }^{4}$ Universidade Federal do Recôncavo da Bahia, UFRB, Brasil. E-mail: < fragmentoflorestal@hotmail.com>.

${ }^{5}$ Universidade do Estado de Santa Catarina, UDESC, Brasil. E-mail: < phiguchi@cav.udesc.br> e <carol_sil4@yahoo.com.br>.

${ }^{6}$ Universidade Federal de Minas Gerais, UFMG, Brasil. E-mail: <ary@icb.ufmg>.
} 
Copaifera langsdorffii, Persea major and Cassia ferruginea) except Calycorectes acutatus, present bigger diameters, and their individual adults predominate in the forest canopy, increasing the basal area and presenting a density of smaller individuals in the lower diametric classes. In the evaluated interval, only $\boldsymbol{D}$. cuneatus altered their distribution pattern (Group1-Group2), while the others increased. In Group1 the main dispersion syndrome is anemochory or autochory, while in Group2 the zoochory syndrome predominates. As the fragment presents a small area, elongated form and semi-urban location associated to a regime of intense disturbances, which may have caused a decrease of the fauna disperseres, compromising the recruitment rate.

Keywords:Tropical semideciduous forest, Community of trees and shrubs, Forest dynamic and Diametric structure.

\section{INTRODUÇÃO}

Na região do Alto Rio Grande, Sul do Estado de Minas Gerais, o processo de alteração das paisagens remete ao início do século XVII (OLIVEIRA-FILHO e MACHADO, 1993). Derivados da floresta primordial, os fragmentos de florestas semideciduais da região foram drasticamente reduzidos, uma vez que sua ocorrência coincidia com solos mais férteis e úmidos que são mais atraentes para a agropecuária (OLIVEIRAFILHO et al., 1994). Tais processos resultaram no isolamento e redução do número de habitats disponíveis nas áreas remanescentes, bem como no aumento da suscetibilidade dessas a diversos fatores impactantes, como a invasão por gado, incêndios, extração seletiva e deposição de lixo, entre outros, o que contribui para a descaracterização fitofisionômica (PEREIRA et al., 2007) e pode culminar na defaunação, a qual consiste na diminuição da riqueza, diversidade e, ou, biomassa de animais (REDFORD, 1992). No entanto, os pequenos fragmentos florestais dispersos pela paisagem, em muitas regiões do Brasil, representam, talvez, a única forma de conservação disponível atualmente, embora sejam os mais sensíveis aos efeitos da fragmentação florestal, estando mais suscetíveis à degradação.

Em ambientes tropicais, o histórico de perturbação tem sido reportado como um dos principais elementos que determinam o processo de dinâmica e a estrutura de comunidades de espécies arbóreas. O entendimento da dinâmica florestal depende de diversas informações fundamentais, podendo ser destacada a avaliação de crescimento por meio das observações dos incrementos em diâmetro, altura e em área basal em determinado intervalo de tempo. Grande parte da estrutura de uma floresta pode ser explicada por meio da avaliação de sua distribuição diamétrica, a qual é definida pela caracterização do número de indivíduos por unidade de área e por intervalo de classe de diâmetro.
Assim. a análise da distribuição de diâmetros permite a realização de inferências sobre o passado e o futuro das comunidades e populações arbóreas. Dessa forma, o objetivo deste trabalho foi verificar as variações no decorrer de cinco anos das classes diamétricas da comunidade e das populações mais abundantes de um remanescente florestal, visando orientar medidas de seu manejo e conservação.

\section{MATERIAL E MÉTODOS}

\section{1. Área de estudo}

Este estudo foi conduzido em um fragmento florestal conhecido como Mata da Lagoa, Lavras, Minas Gerais, nas coordenadas de $21^{\circ} 13^{\prime} 11^{\prime \prime S}$ e $44^{\circ} 58^{\prime} 15^{\prime \prime} \mathrm{W}$, com altitudes variando entre 860 e $890 \mathrm{~m}$. A área total era de cerca de 5,4 ha até 1986, quando sofreu corte raso nas áreas da borda norte, o que reduziu sua área em 25,7\%, chegando aos atuais 4,0 ha (Figura 1). A vegetação se classifica como Floresta Estacional Semidecidual Montana (VELOSO et al., 1991). A área se encontra isolada de outros fragmentos por distâncias superiores a 1 km e está circundada por uma matriz de baixa biomassa, com pastagens e o canal de drenagem de uma represa. Há sinais de corte seletivo de madeira e da penetração do gado.

O clima é do tipo Cwb de Köppen (mesotérmico com verões brandos e suaves e estiagens de inverno). A precipitação e a temperatura média anual são de 1.493,2 mm e $19,3{ }^{\circ} \mathrm{C}$, respectivamente, com $66 \%$ da precipitação ocorrendo de novembro a fevereiro (VILELA e RAMALHO, 1979). Os solos foram classificados, segundo o Sistema Brasileiro de Classificação de Solo da Embrapa (1999), em duas classes: Argissolos Vermelho-Amarelos Distróficos (PVAd) e Nitossolos Vermelhos Eutroférricos (NVef) (MACHADO et al., 2004). 


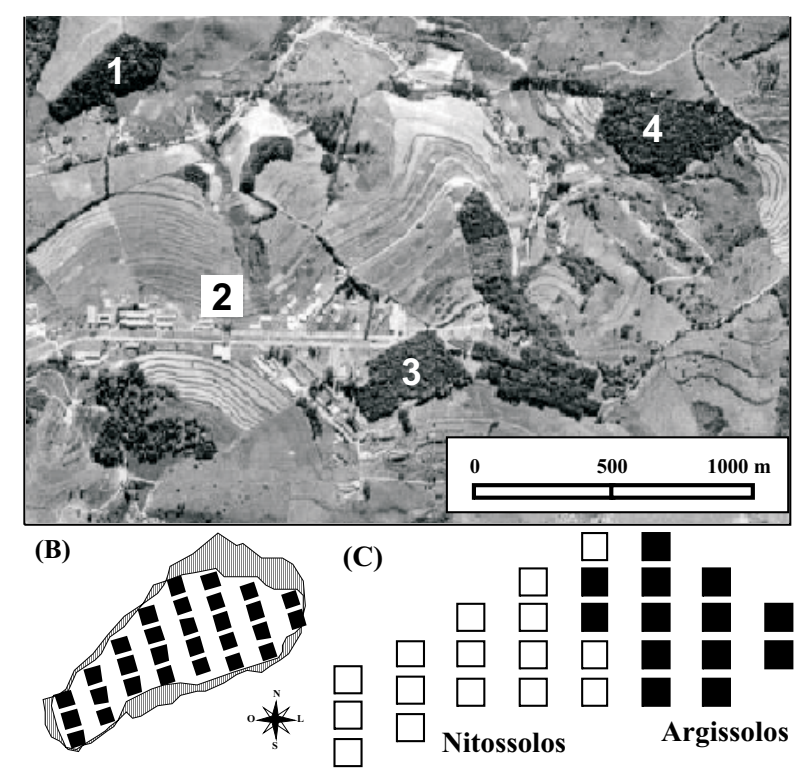

Figura 1 - (A) Foto aérea da área de estudo destacando o fragmento florestal da Mata da Lagoa (1), o Campus da UFLA (2) e os fragmentos mais próximos: a Reserva Florestal da UFLA (3) e a Mata da Subestação (4). (B) Disposição das parcelas (quadrados negros) na Mata da Lagoa e áreas que sofreram corte raso nos últimos 15 anos (hachurado). (C) Classes de solos das parcelas.

Figure 1 - (A) Aerial photograph of the study area highlighting the Mata da Lagoa forest fragment (1), the campus of UFLA (2) and the nearest forest fragments: the Reserva Florestal da UFLA (3) and the Mata da Subestação (4). (B) Distribution of sample plots (black squares) on the Mata da Lagoa and forest areas cleared in the last 15 years (hatched). (C) Soil classes of sample plots.

\subsection{Inventários do compartimento arbóreo-arbustivo}

No ano de 2000 foram alocadas 29 parcelas de $20 \times 20 \mathrm{~m}$, totalizando uma área amostral de 1,16 ha e perfazendo $29,07 \%$ da área total. As parcelas foram distribuídas sistematicamente em oito transeções dispostas de forma a transpor perpendicularmente o fragmento em seu sentido de maior declividade, indo de uma borda à outra. As distâncias foram de $30 \mathrm{~m}$ entre transecções e 10 m entre parcelas. Nessa ocasião, todos os indivíduos arbóreos vivos encontrados nas parcelas com diâmetro à altura do peito (DAP) $\geq 5$ $\mathrm{cm}$ foram marcados. Para cada indivíduo amostrado foi mensurada a circunferência à altura do peito (CAP) com fita métrica e estimada a altura por comparação com o podão de 13 m. A identificação das espécies foi realizada conforme descrito em Machado et al. (2004). Um segundo inventário da comunidade arbórea foi realizado em 2005, utilizando-se os mesmos critérios do anterior. Desse modo, os novos indivíduos que atingiram o critério de inclusão (recrutas) foram marcados e medidos, os mortos foram registrados e os sobreviventes mensurados novamente.

\subsection{Distribuições diamétricas da comunidade e das populações}

Foram preparadas distribuições de densidades de árvores por classes de diâmetros tanto para a comunidade quanto para as 15 espécies de maior valor de importância (VI). A dinâmica das árvores por classe de diâmetro foi analisada empregando-se os mesmos intervalos de classe com amplitudes crescentes para compensar o forte decréscimo da densidade nas classes de tamanhos maiores, típico da distribuição em exponencial negativo, conhecida como $J$-invertido (BOTREL et al., 2002). Esses intervalos permitem melhor representação das classes diamétricas maiores e de baixa densidade, o que é desejável em comparações gráficas (OLIVEIRA-FILHO et al., 2001).

Para descrever as variações temporais ocorridas em cada classe de diâmetro, foi contabilizado o número de árvores que passou pelos seguintes eventos: permanência na classe, morte, recrutamento, imigração (ingrowth) e emigração (outgrowth) na classe, podendo os dois últimos ser progressivos ou regressivos (LIEBERMAN et al., 1985). Com base na distribuição de diâmetros em 2000, foram utilizados testes de qui-quadrado para verificar se as frequências de árvores vivas em 2005 e de árvores mortas foram independentes das classes de diâmetro. A diferença entre o número de árvores ingressantes (recrutas + imigrantes) e árvores egressas (mortos + emigrantes) em cada classe de diâmetro foi verificada por comparações entre contagens de Poisson para a amostra total e para as populações estudadas.

\section{RESULTADOS E DISCUSSÃO}

A distribuição nas classes diamétricas da comunidade arbórea apresentou, em ambos os inventários (2000 e 2005), claro padrão de exponencial negativo ( $J$-invertido), ou seja, alta concentração de indivíduos nas classes menores e redução acentuada no sentido das classes maiores (Figura 2). A classe

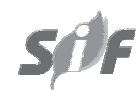

Revista Árvore, Viçosa-MG, v.34, n.4, p.723-732, 2010 


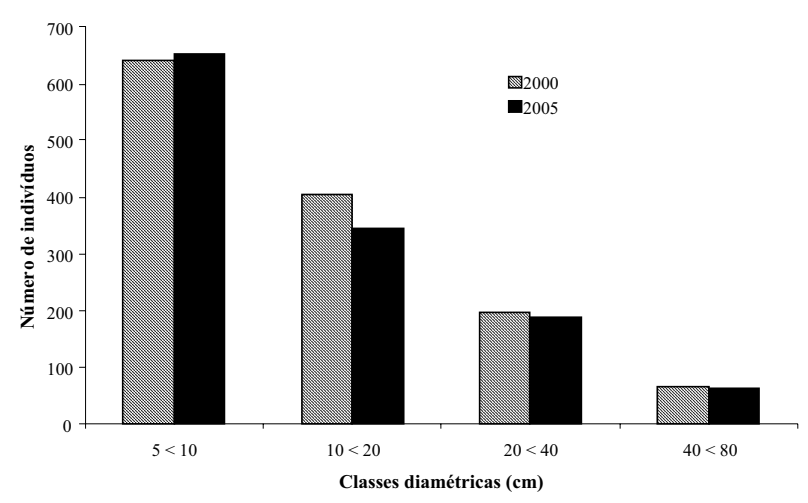

Figura 2 - Distribuição por classe de diâmetro do número de indivíduos arbóreo-arbustivos inventariados na Mata da Lagoa, Lavras, MG.

Figure 2-Distribution per diameter class of the number of individual trees and shrubs surveyed in the Mata da Lagoa, Lavras, SE Brazil.

de DAP entre 5 e $10 \mathrm{~cm}$ compreendeu 49,0\% e 52,3\% (2000 e 2005, respectivamente) dos indivíduos, demonstrando que a importância relativa dessa classe se acentuou no período analisado, embora essa variação não tenha sido significativa ( $t=-0,830 ; p=0,4138)$. Na classe consecutiva, 10,1 a $20 \mathrm{~cm}$, foi verificado padrão inverso, ou seja, em 2000 essa classe contribuía com 31,0\%, enquanto em 2005, com 27,6\%; no intervalo analisado houve redução significativa ( $t=3,958 ; p=0,0004$ ) na participação dessa classe na estrutura florestal. As classes maiores, 20,1 a $40 \mathrm{~cm}$ e 40,1 a $80 \mathrm{~cm}$, não apresentaram mudanças significativas, compreendendo $15,0 \%$ na terceira classe ( $t=0,927 ; p=0,3615$ ) em ambos os inventários e na quarta classe, 4,9\% e 5,0\%, 2000 e 2005, respectivamente $(t=0,494 ; p=0,6256)$.

Muito embora quantidade elevada de indivíduos nas classes inferiores possa sugerir que as espécies estarão garantidas na estrutura futura da floresta, a distribuição diamétrica é um reflexo das características autoecológicas das espécies, ou seja, não é o fato de possuir maior densidade que as espécies irão garantir sua manutenção na comunidade, mas sim sua capacidade de competir dentro do seu nicho ecológico (SCHAAF et al., 2006). Dessa forma, a maior densidade de indivíduos menores deve ser considerada com cautela, demonstrando a necessidade de análise mais detalhada, no nível específico e com um grupo maior de espécies para permitir interpretações mais seguras das distribuições diamétricas (SANTOS et al., 1998).
A quase totalidade dos inventários de comunidades arbóreo-arbustivas de florestas autóctones apresentou distribuição diamétrica seguindo o modelo $J$-invertido, ou exponencial negativo. Entretanto, quando foram analisadas as espécies isoladamente, observou-se que somente algumas delas seguiam o mesmo padrão da comunidade(CARVALHO et al., 1995; OLIVEIRA-FILHO et al., 1994). Felfili (1993) comentou que essas variações são geralmente relacionadas à ecologia populacional de cada espécie e que, na maioria dos casos, o que se verificou foi que havia grandes descontinuidades ou achatamentos nas distribuições, chegando até a ausência quase total de indivíduos jovens em algumas espécies.

Nesse caso, quando foram analisadas as distribuições diamétricas das espécies de maior valor de importância, com base no primeiro inventário (2000), notou-se que elas apresentaram realmente padrões distintos, que foram arbitrariamente distribuídos em dois grupos. O Grupo 1 (Figura 3) era formado predominantemente por espécies de menor porte e abundantes no sub-bosque, como Galipea jasminiflora, Allophylus edulis, Sebastiania commersoniana, Dendropanax cuneatus e Mollinedia widgrenii, mas também incluía duas espécies de árvores altas e caráter pioneiro, Acacia polyphylla e Piptadenia gonoacantha. E o Grupo 2 (Figura 4) era composto pelas espécies Platycyamus regnellii, Calycorectes acutatus, Cupania vernalis, Machaerium stipitatum, Machaerium villosum, Copaifera langsdorffii, Persea major e Cassia ferruginea. Todas, exceto C. acutatus, eram espécies de maior porte, cujos indivíduos adultos predominavam no dossel da floresta, contribuindo, assim, para elevar a área basal.

As distribuições diamétricas do Grupo 1 seguiam o modelo exponencial negativo com concentração de indivíduos na classe de menor diâmetro, enquanto as espécies do Grupo 2 apresentaram como característica comum densidade de indivíduos da menor classe diamétrica menor do que a da classe subsequente. Algumas espécies como P. regnellii resultaram em distribuições unimodais e outras, como M. villosum, em distribuições com densidade crescente na direção das classes de maior diâmetro. Esses padrões se mostraram consistentes em ambos os inventários. A única espécie que apresentou mudança significativa nos padrões foi $D$. cuneatus, em que no primeiro inventário apresentou distribuição do tipo exponencial negativo e, no segundo levantamento, a tendência foi de distribuição normal. 

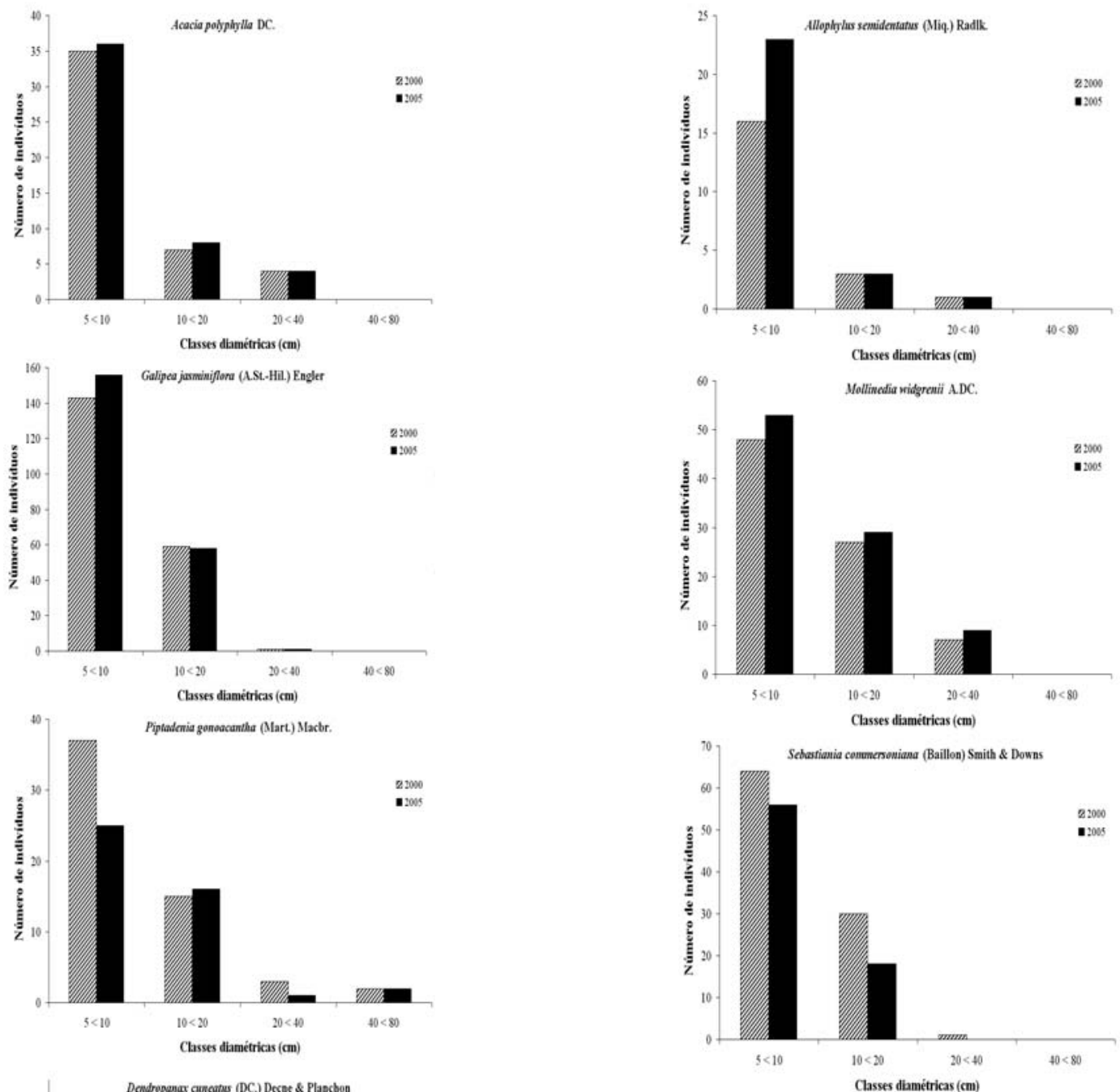

Classes thiamétricas (cm)

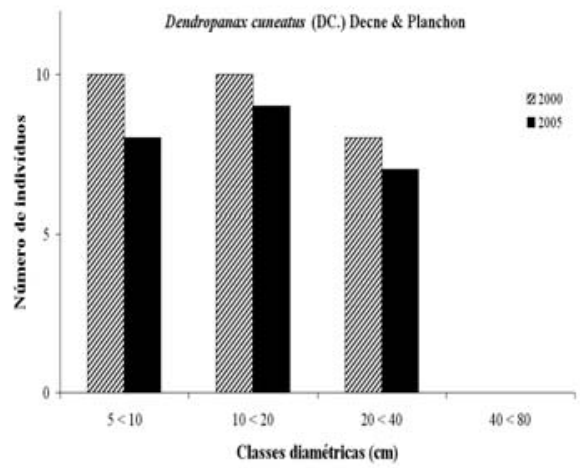

Figura 3 - Distribuição por classe de diâmetro do número de indivíduos arbóreo-arbustivos das sete espécies inventariadas na Mata da Lagoa, Lavras, MG, e pertencentes ao Grupo 1 (ver texto).

Figure 3 - Distribution per diameter class of the number of individual trees and shrubs of the seven species inventoried in the Mata da Lagoa, Lavras, SE Brazil, and belonging to Group 1 (see text). 

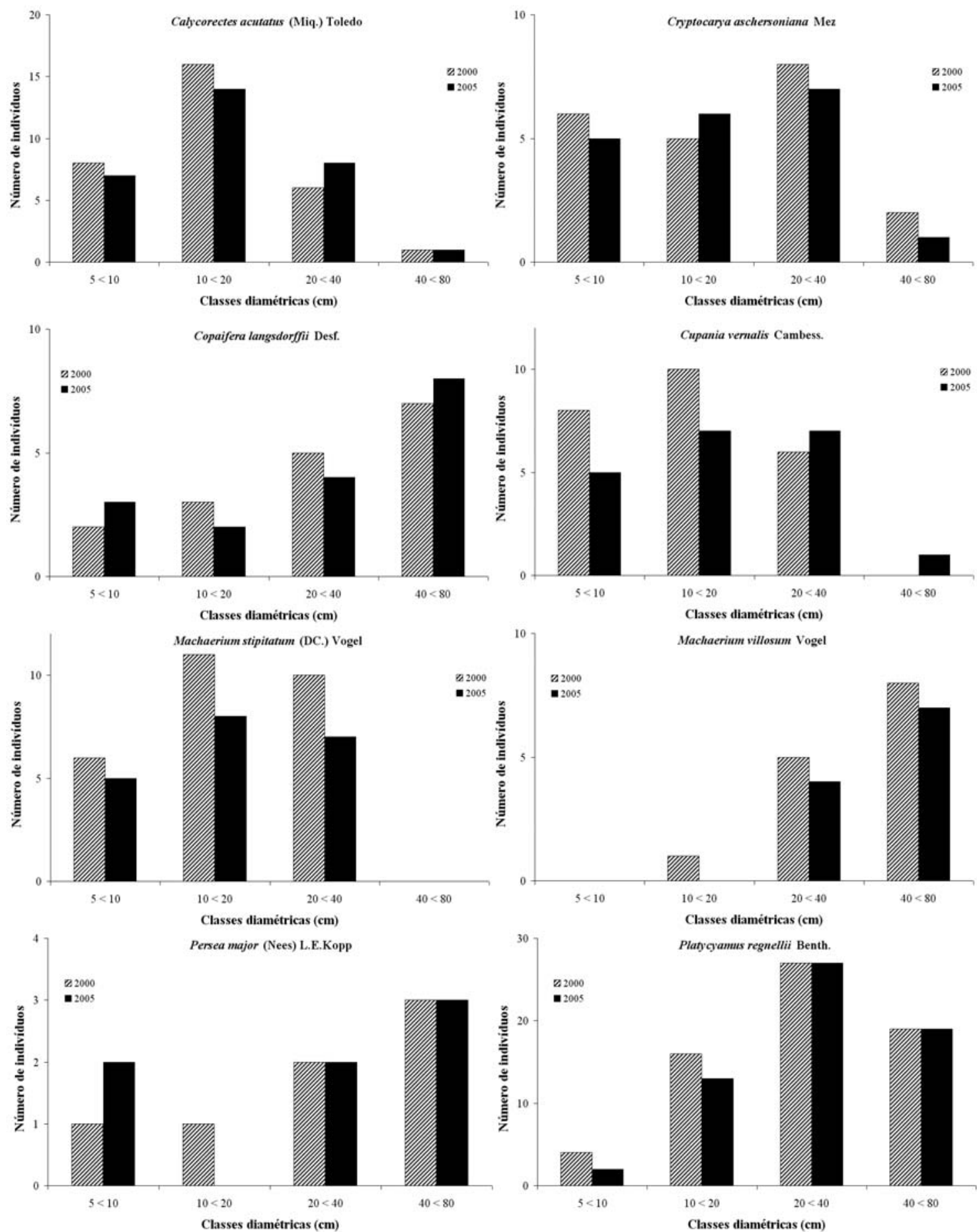

Figura 4 - Distribuição por classe de diâmetro do número de indivíduos arbóreo-arbustivos das oito espécies inventariadas na Mata da Lagoa, Lavras, MG, e pertencentes ao Grupo 2 (ver texto).

Figure 4 - Distribution per diameter class of the number of individual trees and shrubs of the eight species inventoried in the Mata da Lagoa, Lavras, SE Brazil, and belonging to Group 2 (see text).

Revista Árvore, Viçosa-MG, v.34, n.4, p.723-732, 2010 
As diferenças entre os dois grupos podem estar relacionadas a diversos fatores, incluindo aspectos da história natural de cada espécie e do histórico de perturbações do fragmento. No Grupo 1, o estoque expressivo de indivíduos menores pode simplesmente resultar da menor amplitude de tamanhos das quatro espécies de sub-bosque. Contudo, a abundância de indivíduos de pequeno porte das duas espécies de grandes árvores pioneiras sugeriu que o regime de perturbações é relativamente intenso e contínuo.

A relativa escassez de indivíduos de pequeno porte nas oito espécies do Grupo 2 indicou alguma forma de limitação ao recrutamento dessas, resultando em relativa dificuldade de formar expressivo banco de imaturos no sub-bosque. É importante salientar que foram registradas em dois outros fragmentos florestais da região distribuições diamétricas exponenciais negativas nas seis das oito espécies do Grupo 2, a saber: C. langsdorffii, C. aschersoniana, P. pyrifolia, C. vernalis, $M$. villosum e $M$. stipitatum (CARVALHO et al., 1995; OLIVEIRA-FILHO et al., 1994). Assim, esse padrão de distribuição unimodal pode estar evidenciando fuga dos polinizadores e dispersores da área, visto que seis espécies do Grupo 2 (exceto P. regnellii, $M$. villosum e $M$. stipitatum) apresentaram síndrome de dispersão zoocórica.

O fato de o fragmento florestal ter pequena área, forma alongada e localização semiurbana associada a um regime de intensas perturbações pode ter ocasionado empobrecimento da fauna dispersora, consequentemente prejudicando o recrutamento de indivíduos nas classes de menor diâmetro. D’Angelo Neto et al. (1998), trabalhando com fragmentos próximos à Mata da Lagoa, registraram significativa diminuição da diversidade da avifauna nos fragmentos mais perturbados e de menor área.

A diminuição e isolamento das áreas, bem como a presença de impactos (corte seletivo, fogo e outros), promovem alterações drásticas na estrutura desse remanescente, resultando em empobrecimento na riqueza e abundância de indivíduos, bem como na estrutura de guildas de animais, conforme é amplamente documentado na literatura a respeito de aves (BORGES e GUILHERME, 2000; CÂNDIDO JUNIOR, 2000; GUILHERME E CINTRA, 2001; SANTOS, 2004; CINTRA e SANAIOTTI, 2005; CINTRA et al., 2006), morcegos (FLEMING e HEITHAUS, 1981; SCHULZE et al., 2000;
REIS et al., 2003), pequenos mamíferos (ALHO, 1981; PARDINI e UMETSU, 2006) e primatas (CHAPMAN, 1995; VIDAL e CINTRA, 2006). Esses animais têm importantes papéis na manutenção das florestas, de modo que sua extinção local pode ter sérias consequências na estrutura e funcionamento da vegetação. Esse fenômeno é conhecido como "síndrome da floresta vazia” (REDFORD, 1992), a qual nem sempre é tão visível como a fragmentação florestal.

A ausência de animais dispersores de propágulos pode afetar tanto a quantidade da dispersão, pela redução no número de sementes dispersas, quanto a qualidade da dispersão, a qual pode ocorrer se os dispersos substitutos manipulam ou depositam as sementes em locais inapropriados para o seu sucesso na germinação e estabelecimento (CORDEIRO e HOWE, 2001).

Além da diminuição na área disponível e do aumento do grau de isolamento, a fragmentação pode diminuir a heterogeneidade de habitats locais e gerar a deterioração dos habitats remanescentes ao longo do tempo (SAUNDERS et al., 1991; ANDRÉN, 1994; LAURANCE et al., 2002). Tais fatores, além de variarem com o tamanho da área e o grau de isolamento, variam com o formato do remanescente e com o uso que é feito das áreas do seu entorno (SAUNDERS et al., 1991).

\section{CONSIDERAÇÕES FINAIS}

Os padrões encontrados sugerem forte degradação do fragmento, que pode resultar em processos de endogamia e extinção local. Esses resultados se tornam ainda mais relevantes, visto que esses padrões foram detectados exatamente nas populações de espécies dominantes, as quais são reconhecidas por terem profundo impacto na composição, dinâmica e funcionamento dos ecossistemas. Assim, tais informações são ainda mais importantes devido à realidade atual de muitas das regiões do Brasil, onde pequenas unidades de habitat representam talvez a única forma de conservação disponível de algo semelhante ao que foram as comunidades primitivas. Isso reforça a convicção de que os pequenos fragmentos florestais dispersos pela paisagem refletem de maneira pálida e imperfeita notável heterogeneidade ambiental, tanto no espaço quanto no tempo, e merecem especial atenção para a compreensão dos processos de manutenção e sustentabilidade da biodiversidade.

Revista Árvore, Viçosa-MG, v.34, n.4, p.723-732, 2010 


\section{REFERÊNCIAS}

ALHO, C. J. R. Small mammal populations of Brazilian cerrado: the dependence of abundance and diversity on habitat complexity. Revista Brasileira de Biologia, v.41, n.2, p.223-230, 1981.

ANDRÉN, H. Effects of habitat fragmentation on birds and mammals in landscapes with different proportions of suitable habitat: a review. Oikos, v. 71, n.2, p.355-366, 1994.

BORGES, S. H.; GUILHERME, E. Comunidade de aves em um fragmento florestal urbana em Manaus, Amazonas, Brasil. Ararajuba, v.8, n.1, p.17-23, 2000.

BOTREL, R. T. et al. Composição florística e estrutura da comunidade arbórea de um fragmento de floresta estacional semidecidual em Ingaí, MG, e a influência de variáveis ambientais na distribuição das espécies. Revista Brasileira de Botânica, v.25, n.2, p.195-213, 2002.

CÂNDIDO JUNIOR, J. F. The edge effect in a forest bird community in Rio Claro, São Paulo State, Brazil. Ararajuba, v.8, n.1, p.9-16, 2000.

CARVALHO, D. A. et al. Estrutura diamétrica e vertical de uma floresta ripária no Alto Rio Grande (Bom Sucesso - Estado de Minas Gerais).

Revista Árvore, v.19, n.4, p.572-586, 1995.

CHAPMAN, C. A. Primate seed dispersal: coevolution and conservation implications. Evolutionary Anthropology, v.4, n.1, p.77-82, 1995.

CINTRA, R.; MARUOKA, A. E.; NAKA, L. N. Abundance of two Dendrocincla woodcreepers (Aves: Dendrocolaptidae) in relation to forest structure in Central Amazonia Acta

Amazonica, v.36, n.2, p.209-220, 2006.

CINTRA, R.; SANAIOTTI, T. M. Fire effects on the composition of a bird community in an Amazonian Savanna (Brazil). Brazilian Journal of Biology, v.65, n.4, p.683-695, 2005.

CORDEIRO, N. J. \& HOWE, H. F. Low recruitment of trees dispersed by animals in African forest fragments. Conservation Biology, v.15, n.6, p.1733-1741, 2001.

Revista Árvore, Viçosa-MG, v.34, n.4, p.723-732, 2010
D’ANGELO NETO, S. et al. Avifauna de quatro fisionomias florestais de pequeno tamanho (5-8 ha) no campus da UFLA. Revista Brasileira de Biologia, v.58, n.3, p.531-549, 1998.

\section{EMPRESA BRASILEIRA DE PESQUISA} AGROPECUÁRIA - EMBRAPA. Centro Nacional de Pesquisa de Solos. Sistema brasileiro de classificação de solos. 2.ed. Rio de Janeiro, 1999.

FELFILI, J. M. Structure and dynamics of gallery forest in Central Brazil. 1993. 125f. Tese (Doutorado em Ecologia) - Oxford University, Oxford, 1993.

FLEMING, T. H. \& HEITHAUS, E. R. Frugivorous bats, seed shadows, and the structure of tropical forests. Biotropica, v.13, (Supl.), p.45-53, 1981.

GUILHERME, E.; CINTRA, R. Effects of intensity and age of selective logging and tree girdling on an understory bird community composition in Central Amazonia, Brazil. Ecotropica, v.7, n.1, p.77-92, 2001.

LAURANCE, W. F. et al. Ecosystem decay of Amazonian forest fragments: a 22-year of investigation. Conservation Biology, v.16, n.3, p.605-618, 2002.

LIEBERMAN, D. et al. Mortality patterns and stand turnover rates in a wet tropical forest in Costa Rica. Journal of Ecology, v.73, n.5, p.915-924, 1985.

MACHADO, E. L. M. et al. Análise comparativa da estrutura e flora do compartimento arbóreoarbustivo de um remanescente florestal na fazenda Beira Lago, Lavras, MG. Revista Árvore, v.28, n.3, p.493-510, 2004.

OLIVEIRA-FILHO, A. T. et al. Variation in tree community composition and structure with changes in soil properties within a fragment of semideciduous forest in southeastern Brazil. Edinburgh Journal of Botany, v.58, n.1, p.139-158, 2001.

OLIVEIRA-FILHO, A. T.; MACHADO, J. N. M. Composição florística de uma floresta semidecídua montana, na Serra de São José, Tiradentes, Minas Gerais. Acta Botanica Brasílica, v.7, n.2, p.71-88, 1993. 
OLIVEIRA-FILHO, A. T.; SCOLFORO, J. R. S.; MELLO, J. M. Composição florística e estrutura de um remanescente de floresta semidecidual montana em Lavras, MG. Revista Brasileira de Botânica, v.17, n.2, p.167-182, 1994.

PARDINI, R. \& UMETSU, F. Pequenos mamíferos não-voadores da Reserva Florestal do Morro Grande - distribuição das espécies e da diversidade em uma área de Mata Atlântica. Biota Neotropica, v.6, n.2, p.1-22, 2006.

PEREIRA, J. A. A.; OLIVEIRA-FILHO, A. T.; LEMOS FILHO, J. P. Environmental heterogeneity and disturbance by humans control much of the tree species diversity of Atlantic montane forest fragments in SE Brazil. Biodiversity and Conservation, v.16, n.4, p.1761-1784, 2007.

REDFORD, K. H. The empty forest. BioScience, v.42, n.6, p.412-422, 1992.

REIS, N. R. et al. O que é melhor para manter a riqueza de espécies de morcegos (Mammalia, Chiroptera): um fragmento florestal grande ou vários fragmentos de pequeno tamanho? Revista Brasileira de Zoologia, v.20, n.2, p.225-230, 2003.

SANTOS, A. M. R. Comunidade de aves em remanescentes florestais secundários de uma área rural no sudeste do Brasil. Ararajuba, v.12, n.1, p.41-49, 2004.

SANTOS, F. A. M. et al. Structure and dynamics of tree species of the Atlantic Forest. Anais da Academia Brasileira de Ciências, v.70, n.4, p.874-880, 1998.
SAUNDERS, D. A.; HOBBS, R. J.; MARGULES, C. R. Biological consequences of ecosystem fragmentation: a review. Conservation Biology v.5, n.1, p.18-32, 1991.

SCHAAF, L. B. et al. Alteração na estrutura diamétrica de uma Floresta Ombrófila Mista no período entre 1979 e 2000. Revista Árvore, v.30, n.2, p.283-295, 2006.

SCHULZE, M. D.; SEAVY, N. E.; WHITACRE, D. F. A comparison of the phyllostomid Bat assemblages in un-disturbed neotropical forest and in forest fragments of a slash-and-burn farming mosaic in Petén, Guatemala.

Biotropica, v.32, n.1, p.174-184, 2000.

VELOSO, H. P.; RANGEL FILHO, A. L. R.; LIMA, J. C. A. Classificação da vegetação brasileira adaptada a um sistema universal. Rio de Janeiro: Instituto Brasileiro de Geografia e Estatística, 1991.

VIDAL, M. D.; CINTRA, R. Effects of forest structure components on the occurrence, group size and density of groups of bare-face tamarin (Saguinus bicolor - Primates: Callitrichinae) in Central Amazonia. Acta Amazonica, v.36, n.2, p.237-248, 2006.

VILELA, E. A.; RAMALHO, M. A. P. Análise das temperaturas e precipitações pluviométricas de Lavras, Minas Gerais. Ciência e Prática, v.3, n.1, p.71-79, 1979. 
